\title{
The Discussion of Multisensor Time Registration Algorithm
}

\author{
Hanlong Chen ${ }^{1, a, *}$, Danhui Sun ${ }^{1, b}$, Jieheng Geng ${ }^{1, c}$, Caikun Zhang ${ }^{1, \mathrm{~d}}$ \\ ${ }^{1}$ Luoyang Electronic Equipment Test Center, Luoyang 471000

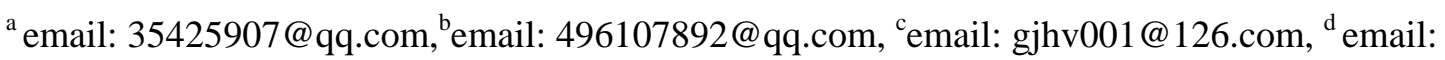 \\ zhangcaikun@foxmail.com \\ * corresponding author
}

Keywords: time registration, multiple sensor, data fusion, simulation analysis

\begin{abstract}
Before data fusion, the center needs to do the work of time registration of the same target data from different sensors in order to control the state of the target in the air in real time. At present, the commonly used time registration algorithm has some methods, including curve fitting method, the Lagrange interpolation method and spline interpolation method, etc. The above three algorithms are mainly introduced in this paper and the author of this paper carries on the simulation study of the three methods. The results of the simulation study verify the effectiveness of the algorithm, and at the same time the author makes comparative analysis on the advantages and disadvantages of these algorithms under different mobile case, which can be used for reference for practical engineering applications.
\end{abstract}

\section{Introduction}

In order to adapt the environment of the more and more complicated modern battlefield and improve the accuracy of the information detection, Americans in the 1970s are the first to put forward the concept of multi-sensor data fusion. According to the measurement results of each sensor, the multi-sensor data fusion system carries through the optimization process in terms of certain rules and methods, and overcomes the shortcomings and limitations of the ability and performance of the single sensor detection so as to get more accurate information and obtain the description of the goals. Currently the definition with high approval is put forward by the Joint Directors of Laboratories (JDL) of the United States from the aspect of military terms ${ }^{[1-2]}$, that is "data fusion is a kind of multi-level and multi-aspect process, which to correlate and combine the data from multiple sensors and sources so as to improve the accuracy of estimation and identity and to make the timely and complete evaluation of the important degree of the battlefield and threat".

In the multiple sensor fusion system, all the measurement data need to transit to the unified time benchmark. But due to the inherent deviation of each sensor, the measurement error and different sampling frequency, the results of directly data fusion are not ideal and even lost the accuracy of registration. So before data fusion, firstly it should complete the time registration of the data. It is a multi-sensor data error elimination and converting process and the precondition of the proper fusion of multi-sensor system and it directly affects the final performance of a system.

Many domestic and foreign scholars and researchers have proposed many solutions to the problem of time registration. Some scholars such as Wang Baoshu ${ }^{[3]}$ propose to use the method of interpolation and extrapolation to carry on time registration because the calculation is simple and it is applicable to the constant speed and slow change of the target. Blair WD ${ }^{[4]}$, Zhou Rui ${ }^{[5]}$ come up with the least squares virtual method. This method can synthesize sampling data to a synchronized data, but it has special requirement to the sampling period. It applied to the simple motion model. Liang $\mathrm{Kai}^{[6]}$ makes an intensive study of the spline fitting method based on least squares. It is better for solving the problem of time unsynchronized and it volatilizes a lot in fitting around the two levels. Liu Lisheng ${ }^{[7]}$ puts forward the method of Taylor expansion and by using this method he completes the time registration of the measured data. 
Among the existing data, there are many separate analysis and improved methods about each algorithm, but there are less comparing quantitative analysis about the algorithm. And there are more studies on the uniform motion model, fewer studies on the accelerated motion model. This paper mainly introduces three commonly used algorithms of time registration: (1) The least squares curve fitting method; (2) Lagrange interpolation method; (3) Spline interpolation method. Through the establishment of a unified simulation model and project, this paper makes a deep analysis about the three algorithms and verifies the validity of the algorithms. Furthermore, this paper compares the advantages and disadvantages of the algorithms, which have certain significance for practical engineering applications.

\section{Time Registration}

Time registration, in a word, is to acquire the data of multi-sensor at the same time. So, before fusion it is necessary to dispose synchronously, namely unified "time base". In practical multisensor data fusion system, because of the difference of the specific content of the fusion tracking and the different application environment, it has different requirements of time registration. The different capability of the sensor itself may also affect the accuracy and the real-time performance of the registration. So in designing the multi-sensor data fusion system, it should be based on specific circumstances and on the premise of the completion of the requirements of the registration, the ultimate performance of registration should be improved from two aspects: the real-time performance and accuracy.

In general time registration model, the main structure and functions should include: analysis of the observation data, the registration method and the calibration frequency selection, analysis of registration data, prior knowledge and registration requirements, etc ${ }^{[8]}$.

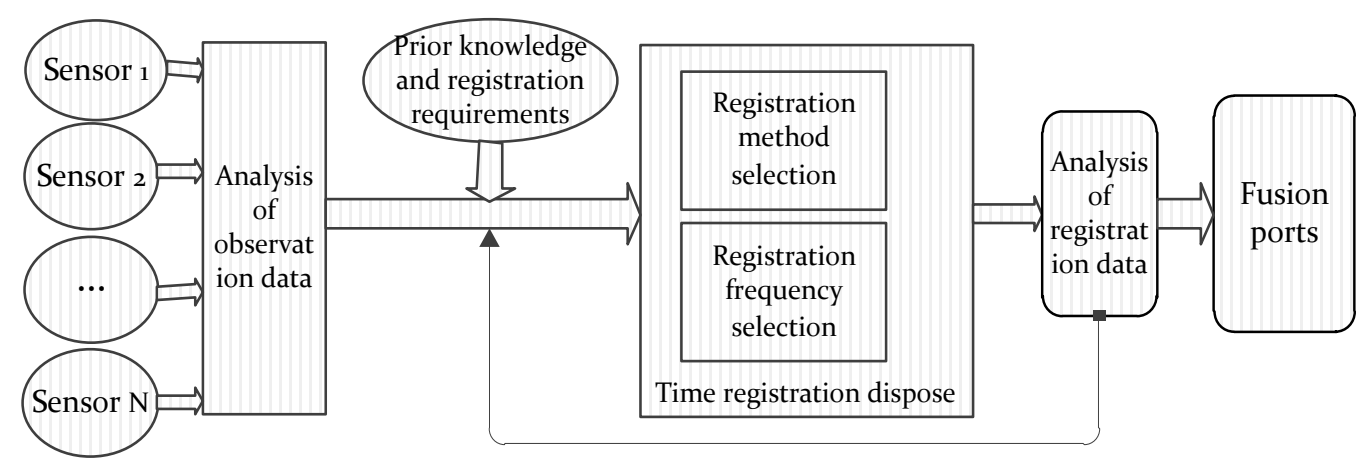

Figure 1 The functional model diagram of time registration

\section{Time registration algorithm}

\subsection{The least squares curve fitting method}

Curve fitting means that to find a curve (expressed in mathematical formula) according to the $\mathrm{n}$ points $\left(x_{i}, y_{i}\right),(i=0,1, \ldots n)$ given in the plane and to make the curve in conformity with these points. The curve represented by the mathematical formula presents approximately the tendency of the data. The above process process is called curve fitting. The curve represented by the curve fitting formula does not necessarily through all the data points and it is just one curve which can better reflect the law of the data.

The Least squares curve fitting used for time registration is based on the least squares principle in keeping the fitting error under the principle of the minimum and to fit the target data of different sampling time, obtaining the approximate curve about the target trajectory, and then realizing the time registration by using the curve equation to calculate target state data of the corresponding registration time. 
If the known data set $\left(\mathrm{x}_{\mathrm{i}}, \mathrm{y}_{\mathrm{i}}\right),(\mathrm{i}=0,1, \ldots \mathrm{n})$, after fitting the curve equation is $\mathrm{P}(\mathrm{x})$, the error $\delta_{\mathrm{i}}$ can be represented as:

$$
\delta_{i}=p\left(x_{i}-y_{i}\right), \quad(i=0,1, \ldots n)
$$

To make data error $\delta_{\mathrm{i}}$ after fitting to the minimum, generally according to the minimum (least squares) discrete error sum of squares method:

$$
\sum_{\mathrm{i}=0}^{\mathrm{n}} \delta^{2}=\min
$$

Fitting function $\mathrm{P}(\mathrm{x})$ is various, usually in the form of a polynomial $\mathrm{P}(\mathrm{x})$, namely to find a function composition $\mathrm{p}(\mathrm{x})=\sum_{\mathrm{j}=0}^{\mathrm{m}}\left(\mathrm{a}_{\mathrm{j}}, \varphi_{\mathrm{j}}(\mathrm{x})\right)$ in the function class $\varphi=\left\{\varphi_{0}, \varphi_{1}, \ldots, \varphi_{\mathrm{m}}\right\}$ to make the error sum of squares $\|\delta\|^{2}$ to the minimum ${ }^{[9]}$ :

$$
\|\delta\|^{2}=\sum_{\mathrm{i}=1}^{\mathrm{n}} \delta^{2}=\sum_{\mathrm{i}=1}^{\mathrm{n}}\left[\mathrm{p}\left(\mathrm{x}_{\mathrm{i}}\right)-\mathrm{y}_{\mathrm{i}}\right]^{2}
$$

Thus, to use the least square method for curve fitting problem is to evaluate $\mathrm{P}(\mathrm{x})$ and to take the minimum of $\|\delta\|^{2}$. So the process of making fitting curve function changes into the evaluation of the minimum of the multivariate function. And the multivariate function is as follows:

$$
\mathrm{I}\left(\mathrm{a}_{0}, \mathrm{a}_{1}, \ldots \mathrm{a}_{\mathrm{m}}\right)=\sum_{\mathrm{i}=1}^{\mathrm{n}} \delta^{2}
$$

After solving the minimum value of multivariate function we can get the least-square solutions of function $\mathrm{p}(\mathrm{x})$ :

$$
\mathrm{p}(\mathrm{x})=\sum_{\mathrm{j}=0}^{\mathrm{n}}\left(\mathrm{a}_{\mathrm{j}} \varphi_{\mathrm{j}}(\mathrm{x})\right)^{2}
$$

Therefor we obtain the fitting curve of the data. In addition, the precision of the fitting curve obtained from different degree of polynomial used for different state of motion is also different. The core of the Least squares curve fitting method is to make multiple measurement data base on least squares criterion fitting for one or several smooth curve by which can we get the measured value of the corresponding moment and obtain data fusion of information then.

\subsection{Lagrange interpolation method}

The Interpolation method is to get the analysis formula based on the known data of the function. It requests that through the known sample points the approximate functions are determined and then calculate the request time data according to functional expression ${ }^{[7]}$. Using the method of data interpolation for multisensory time registration is to obtain the equation of locus of the target motion according to the known sample point data and then obtain the registration time data through the equation.

Lagrange interpolation method is to use Lagrange interpolation polynomial estimation function expressions to compute the data of time registration according to the function expression obtained. Suppose it needs to make time registration on a sensor, we can set $p_{i}=\left(x_{i}, y_{i}, z_{i}\right)$ is the sampling data of the sensor in $t_{i}(i=1 \ldots n)$ and the moment of the time registration is $t_{j}\left(t_{i}<t_{j}<\right.$ $\left.t_{i+1}\right)$, the result after time registration is presented by $\left(x_{i j}, y_{i j}, z_{i j}\right)$, its sampling sequence is as follows:

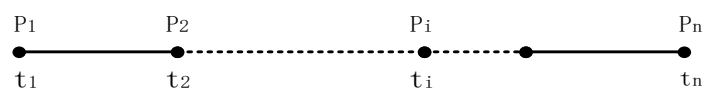

Figure 2 Sampling sequence of a sensor 
Then we can use the Lagrangian linear interpolation method (assuming the target for uniform linear motion), parabolic interpolation method (assuming the target for uniformly accelerated motion) and the high order interpolation method (assuming the target trajectory for high order polynomial curve) for registration. Here, we mainly discuss the acceleration and varying accelerated motion of Lagrange interpolation method.

Lagrange parabola interpolation method (i.e. the Lagrange interpolation method), assumes that the target is of uniformly accelerated motion model, and the interpolation formula is as follows:

$$
\begin{aligned}
& L_{x}\left(t_{i j}\right)=\frac{\left(t_{i j}-t_{i-1}\right)\left(t_{i j}-t_{i+1}\right)}{\left(t_{i-1}-t_{i}\right)\left(t_{i-1}-t_{i+1}\right)} x_{i-1}+\frac{\left(t_{i j}-t_{i-1}\right)\left(t_{i j}-t_{i+1}\right)}{\left(t_{i}-t_{i-1}\right)\left(t_{i}-t_{i+1}\right)} x_{i+} \frac{\left(t_{i j}-t_{i-1}\right)\left(t_{i j}-t_{i}\right)}{\left(t_{i+1}-t_{i-1}\right)\left(t_{i+1}-t_{i}\right)} x_{i+1} \\
& L_{y}\left(t_{i j}\right)=\frac{\left(t_{i j}-t_{i-1}\right)\left(t_{i j}-t_{i+1}\right)}{\left(t_{i-1}-t_{i}\right)\left(t_{i-1}-t_{i+1}\right)} y_{i-1}+\frac{\left(t_{i j}-t_{i-1}\right)\left(t_{i j}-t_{i+1}\right)}{\left(t_{i}-t_{i-1}\right)\left(t_{i}-t_{i+1}\right)} y_{i+} \frac{\left(t_{i j}-t_{i-1}\right)\left(t_{i j}-t_{i}\right)}{\left(t_{i+1}-t_{i-1}\right)\left(t_{i+1}-t_{i}\right)} y_{i+1} \\
& L_{z}\left(t_{i j}\right)=\frac{\left(t_{i j}-t_{i-1}\right)\left(t_{i j}-t_{i+1}\right)}{\left(t_{i-1}-t_{i}\right)\left(t_{i-1}-t_{i+1}\right)} z_{i-1}+\frac{\left(t_{i j}-t_{i-1}\right)\left(t_{i j}-t_{i+1}\right)}{\left(t_{i}-t_{i-1}\right)\left(t_{i}-t_{i+1}\right)} z_{i+} \frac{\left(t_{i j}-t_{i-1}\right)\left(t_{i j}-t_{i}\right)}{\left(t_{i+1}-t_{i-1}\right)\left(t_{i+1}-t_{i}\right)} z_{i+1}
\end{aligned}
$$

High Lagrange interpolation method (namely the Lagrange interpolation method more) assumes that the target motion trajectory is high order polynomial curve,and its difference formula is:

$$
\mathrm{L}_{\mathrm{x}}\left(\mathrm{t}_{\mathrm{ij}}\right)=\sum_{\mathrm{i}=0}^{\mathrm{n}}\left(\prod_{\substack{\mathrm{k}=0 \\ \mathrm{k} \neq \mathrm{i}}}^{\mathrm{n}} \frac{\mathrm{t}_{\mathrm{ij}}-\mathrm{t}_{\mathrm{k}}}{\mathrm{t}_{\mathrm{i}}-\mathrm{t}_{\mathrm{k}}}\right) \mathrm{x}_{\mathrm{i}}
$$

The interpolation function of Lagrange interpolation method is a polynomial function. If the number of polynomial is too high, it will make the error of the time registration results becomes bigger. In general, the highest interpolation polynomial function is not higher than six. In the practical engineering application, it needs to choose the appropriate Lagrange interpolation function according to the different state of the motion target.

\subsection{Spline function interpolation method}

Spline function interpolation is to fit data points by using piecewise spline function and eventually form a smooth curve of all data points and then obtain the data of the time according to the curve formula. Using spline function interpolation method for multi-sensor time registration is to obtain a smooth curve through spline interpolation on the known sampling points data and then obtain the data of the time registration through the analytic expression of the curve. In practice, it is more often using the cubic spline function interpolation. The cubic spline function interpolation is to measure the target according to the division of the sampling times and then construct a cubic spline interpolation function according to the given time point and the corresponding observations.

Supposing sensor A measures the target for $\mathrm{n}+1$ times in the time period $[\mathrm{a}, \mathrm{b}]$ and dividing the whole sampling time interval according to the sampling time into $a=t_{0}<t_{1}<\cdots<t_{n}=b$, the sampling data value of the sensor corresponding with the given sampling time $t_{i}$ is $f\left(t_{i}\right)=y_{i}$, (i= $0,1, \ldots \mathrm{n})$, to construct a cubic spline differential function $\mathrm{s}(\mathrm{x})$ and making it meet the following conditions:

(1) $s\left(t_{i}\right)=y_{i}(i=0,1, \ldots n)$;

(2) $s(t)$ is a cubic polynomial between each district $\left[t_{i}, t_{i+1}\right](i=0,1, \ldots n-1)$;

(3) $s(t)$ is a second order continuous reciprocal on [a, b].

The spline function interpolation based on least squares in the spline function space $S_{k}(g)$ finds out the optimal approximation of norm $\|\mathrm{g}\|$ with regard to the function $\mathrm{f}(\mathrm{t})$, namely to find out $\hat{s}(\mathrm{t})$ needs to:

$$
\|f-\hat{\mathrm{s}}\|=\min _{\hat{\mathrm{s}} \epsilon \mathrm{S}_{k}(\mathrm{~g})}\|f-\mathrm{s}\|
$$


The construction of the cubic spline interpolation function process is as follows:

Set $m_{i}=s^{\prime} t_{i}, \quad(i=0,1, \ldots n)$, in each district $\left[t_{i}, t_{i+1}\right]$, set $h_{i}=t_{i+1}-t_{i}$, obtaining the computational formula of the cubic spline interpolation function $s(t)$ by using the Hermite interpolation formulas:

$$
\begin{aligned}
& s(t)=\left(1+2 \frac{t-t_{i}}{h_{i}}\right)\left(\frac{t_{i+1}-t}{h_{i}}\right)^{2} y_{i}+\left(1+2 \frac{t_{i+1}-t}{h_{i}}\right)\left(\frac{t-t_{i}}{h_{i}}\right)^{2} y_{i+1} \\
& +\left(t-t_{i}\right)\left(\frac{t_{i+1}-t}{h_{i}}\right)^{2} m_{i}+\left(t-t_{i+1}\right)\left(\frac{t-t_{i}}{h_{i}}\right)^{2} m_{i+1}
\end{aligned}
$$

Using the third condition: $\mathrm{s}^{\prime \prime}\left(\mathrm{t}_{\mathrm{i}}^{-}\right)=\mathrm{s}^{\prime \prime}\left(\mathrm{t}_{\mathrm{i}}^{+}\right)(\mathrm{i}=0,1, \ldots \mathrm{n})$, and additional boundary condition $s\left(t_{0}\right)=s\left(t_{n}\right)=0$, we can get the equation set:

$$
\left\{\begin{array}{c}
2 \mathrm{~m}_{0}+\mathrm{a}_{0} \mathrm{~m}_{1}=\beta_{0} \\
\left(1-\mathrm{a}_{\mathrm{i}}\right) \mathrm{m}_{\mathrm{i}-1}+2 \mathrm{~m}_{\mathrm{i}}+\mathrm{a}_{\mathrm{i}} \mathrm{m}_{\mathrm{i}+1}=\beta_{\mathrm{i}} \\
\left(1-\mathrm{a}_{\mathrm{n}}\right) \mathrm{m}_{\mathrm{n}-1}+2 \mathrm{~m}_{\mathrm{n}}=\beta_{\mathrm{n}}
\end{array}\right.
$$

Among these, $\mathrm{a}_{0}=1, \mathrm{a}_{\mathrm{i}}=\frac{\mathrm{h}_{\mathrm{i}-1}}{\mathrm{~h}_{\mathrm{i}-1}-\mathrm{h}_{\mathrm{i}}}, \quad \beta_{0}=\frac{3}{\mathrm{~h}_{0}}\left(\mathrm{y}_{1}-\mathrm{y}_{0}\right), \beta_{\mathrm{n}}=\frac{3}{\mathrm{~h}_{\mathrm{n}-1}}\left(\mathrm{y}_{\mathrm{n}}-\mathrm{y}_{\mathrm{n}-1}\right)$.

We can get the following equations:

$$
\left\{\begin{array}{c}
2 \mathrm{~m}_{0}+\mathrm{a}_{0} \mathrm{~m}_{1}=\beta_{0} \\
\left(1-\mathrm{a}_{1}\right) \mathrm{m}_{0}+2 \mathrm{~m}_{1}+\mathrm{a}_{1} \mathrm{~m}_{2}=\beta_{1} \\
\left(1-\mathrm{a}_{2}\right) \mathrm{m}_{1}+2 \mathrm{~m}_{2}+\mathrm{a}_{2} \mathrm{~m}_{3}=\beta_{2} \\
\ldots \ldots \ldots \\
\left(1-\mathrm{a}_{\mathrm{i}}\right) \mathrm{m}_{\mathrm{i}-1}+2 \mathrm{~m}_{\mathrm{i}}+\mathrm{a}_{\mathrm{i}} \mathrm{m}_{\mathrm{i}+1}=\beta_{\mathrm{i}} \\
\ldots \ldots \ldots \\
\left(1-\mathrm{a}_{\mathrm{n}-1}\right) \mathrm{m}_{\mathrm{n}-2}+2 \mathrm{~m}_{\mathrm{n}-1}+\mathrm{a}_{\mathrm{n}-1} \mathrm{~m}_{\mathrm{n}}=\beta_{\mathrm{n}-1} \\
\left(1-\mathrm{a}_{\mathrm{n}}\right) \mathrm{m}_{\mathrm{n}-1}+2 \mathrm{~m}_{\mathrm{n}}=\beta_{\mathrm{n}}
\end{array}\right.
$$

It is known from (2.34) that the coefficient matrix of the equations is the triangular matrix whose determinant is not zero, so the solution of equations is only one. By solving the equation set we can get the recursive formulas:

$$
\mathrm{m}_{\mathrm{i}}=\mathrm{a}_{\mathrm{i}} \mathrm{m}_{\mathrm{i}+1}+\mathrm{b}_{\mathrm{i}}(\mathrm{i}=\mathrm{n}, \mathrm{n}+1, \quad \ldots 0)
$$

Among these: $a_{i}=\frac{-a_{i}}{2+\left(1-a_{i}\right) a_{i-1}}, \quad b_{i}=\frac{\beta_{i}-\left(1-a_{i}\right) b_{i-1}}{2+\left(1-a_{i}\right) a_{i-1}}, \quad a_{0}=-\frac{a_{0}}{2}, \quad b_{0}=-\frac{\beta_{0}}{2} \quad(i=n, n+$ $1, \quad \ldots 0)$

Using the formula to solve $a_{i}, b_{i}$, making $m_{i+1}=0$ to obtain $m_{n}, m_{n-1}, \ldots m_{0}$, and make the given parameters $t_{i}, y_{i}, m_{i}$ substitute into $s(x)$, then we can obtain the cubic spline interpolation function.

The Cubic spline interpolation method solves the problem of time synchronization in data fusion and the inconsistent data rate. It has simple calculation and high speed. But the interpolation function strictly requires going through all the given point, if there are some observation errors in the given data then the interpolation result will keep all the influence of the observational error, which causes that the interpolation function in some cases cannot well reflect the characteristics of the part of the data set.

\section{The simulation experiment}

Suppose that there are two sensors: sensor 1 and sensor 2. The two sensors begin to measure the target at the same time. The sampling period of sensor 1 is $\mathrm{Tl}=2.3 \mathrm{~s}$, the sampling period of sensor 2 is $\mathrm{T} 2=3.5 \mathrm{~s}$, the total sampling time is $100 \mathrm{~s}$, the random noise of the two sensors is white gaussian noise whose average value is 0 and whose variance is 80 . 
Now assumes that the target goes through two kinds of movements: uniformly accelerated motion and variable accelerated motion, it cannot directly fuse the data because of the different sampling period. Now through the above three methods we can transform the target observation data obtained by each data into a unified integration point and using the method of Monte Calrlo (that is, the statistical test method) to do the statistical sampling simulation, and finally calculating the root mean square error of the system and comparing the advantages and disadvantages of each algorithm under different motion state.

\subsection{The time registration under uniformly accelerated motion model}

To set two sensors stand from the same target location and the distance is $\mathrm{x} 0=50 \mathrm{~km}$, under the circumstances of uniformly accelerated motion, the target enters the detection area with the initial speed $\mathrm{v} 0=160 \mathrm{~m} / \mathrm{s}$ and the accelerated speed:a $=3 \mathrm{~m} / \mathrm{s} 2$, then using the above three methods to do the time registration of sensor A and sensor B.

The registration results are as follows:

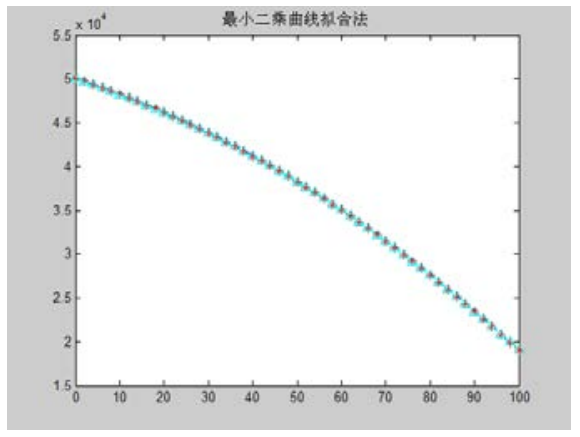

Figure 3 The registration results of least squares curve fitting

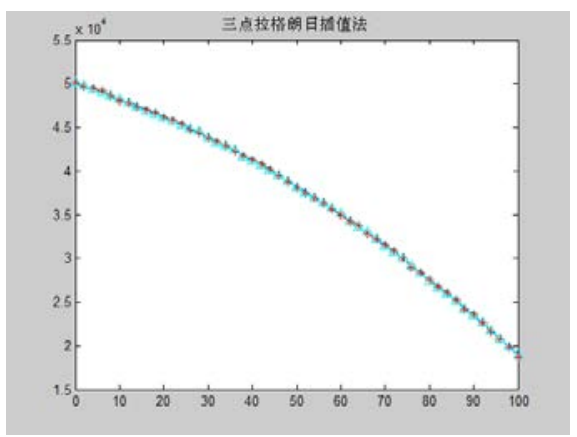

Figure 4 The registration results of Lagrange interpolation method

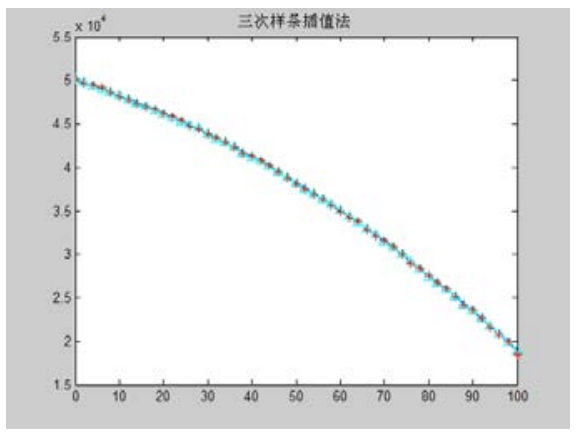

Figure 5 The registration results of Cubic spline interpolation method 


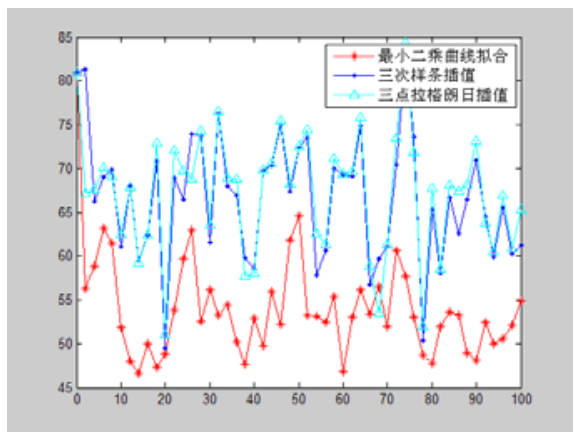

Figure 6 Root mean square error analysis after registration

\subsection{The time registration under varying accelerated motion model}

To set two sensors stand from the same target location and the distance is $\mathrm{x} 0=50 \mathrm{~km}$, under the circumstances of varying accelerated motion, the initial speed of the target is $v 0=160 \mathrm{~m} / \mathrm{s}$, the equation of motion is $x=x_{0}+\frac{v_{0}}{w} \cos w(t), w=\frac{\pi}{20} \mathrm{rad} / \mathrm{s}$ and the observation time is $100 \mathrm{~s}$, then we can do the time registration of sensor $\mathrm{A}$ and sensor $\mathrm{B}$.

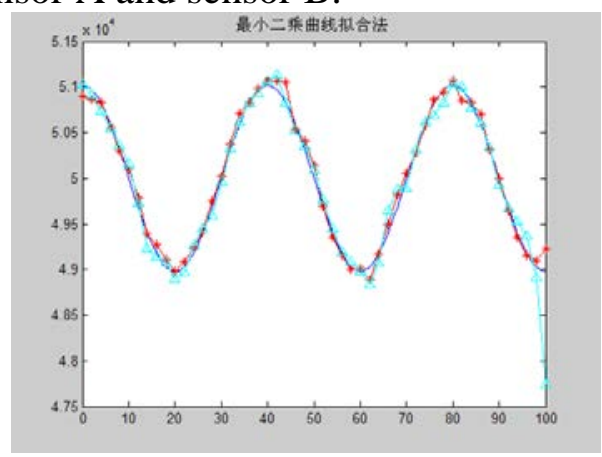

Figure 7 The registration results of least squares curve fitting

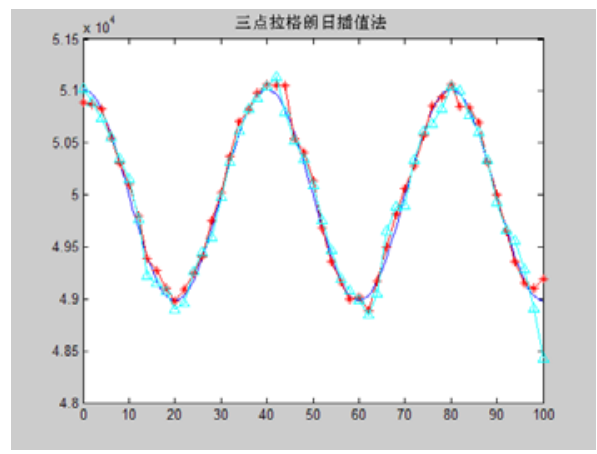

Figure 8 The registration results of Lagrange interpolation method

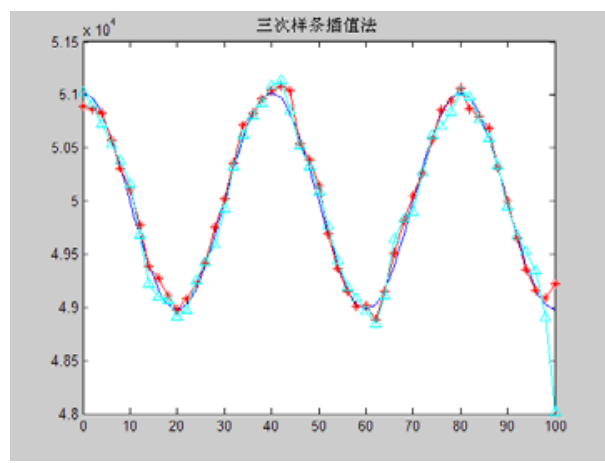

Figure 9 The registration results of Cubic spline interpolation method 


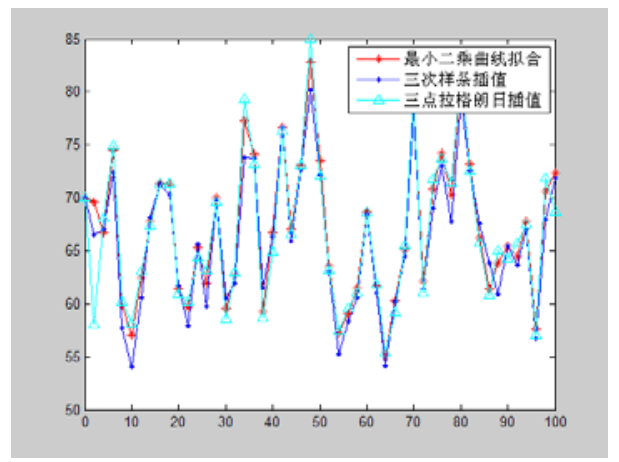

Figure 10 Root mean square error analysis after registration

\section{The simulation analysis}

(1) The simulation result shows that under the uniformly accelerated motion model, all the three kinds of algorithms can effectively complete the registration of various sensors. By comparison, the least squares curve fitting method has more accurate registration performance, while the registration results of the cubic spline interpolation method and Lagrange interpolation method are very close, and the root mean square error of the three kinds of algorithm are as follows:

Table1 The analysis and comparison root mean square error in the system of uniformly accelerated motion

\begin{tabular}{|c|c|c|c|}
\hline method expor data & $\begin{array}{c}\text { The least } \\
\text { squares curve } \\
\text { fitting method }\end{array}$ & $\begin{array}{c}\text { Lagrange } \\
\text { interpolation } \\
\text { method }\end{array}$ & $\begin{array}{c}\text { Cubic spline } \\
\text { interpolation } \\
\text { method }\end{array}$ \\
\hline $\begin{array}{c}\text { Minimum root mean } \\
\text { square error of fitting } \\
\text { points }\end{array}$ & 46,6032 & 51,0113 & 49,4739 \\
\hline $\begin{array}{c}\text { Maximum root mean } \\
\text { square error of fitting } \\
\text { points }\end{array}$ & 80,9200 & 84,3590 & 82,8172 \\
\hline $\begin{array}{c}\text { The average root mean } \\
\text { square error }\end{array}$ & 54,0471 & 67,1676 & 66,6476 \\
\hline
\end{tabular}

The average root mean square error in the system: least squares curve fitting < cubic spline interpolation method $<$ Lagrange interpolation method.

(2) Under the varying accelerated motion model, the three kinds of algorithms can also effectively complete the registration of various sensors. By comparison, the matching performance of the cubic spline interpolation is better and the root mean square errors of the three algorithms are as follows: 
Table 2 The analysis and comparison root mean square error in the system of varying accelerated motion

\begin{tabular}{|c|c|c|c|}
\hline registration & $\begin{array}{c}\text { The least } \\
\text { squares curve } \\
\text { fitting method }\end{array}$ & $\begin{array}{c}\text { Lagrange } \\
\text { interpolation } \\
\text { method }\end{array}$ & $\begin{array}{c}\text { Cubic spline } \\
\text { interpolation } \\
\text { method }\end{array}$ \\
\hline $\begin{array}{c}\text { Minimum root mean } \\
\text { square error of fitting } \\
\text { points }\end{array}$ & 53,7257 & 54,1053 & 52,0519 \\
\hline $\begin{array}{c}\text { Maximum root mean } \\
\text { square error of fitting } \\
\text { points }\end{array}$ & 79,8164 & 79,2130 & 78,4916 \\
\hline $\begin{array}{c}\text { The average root mean } \\
\text { square error }\end{array}$ & 66,2130 & 64,8898 & 64,2257 \\
\hline
\end{tabular}

The average root mean square error in the system: cubic spline interpolation method $<$ Lagrange interpolation method < least squares curve fitting.

\section{Conclusion}

This article gives a brief overview of theories of time registration. It describes the function model of the time registration according to the process and structure of time registration. This paper also introduces the three kinds of algorithms for time registration and carried out the simulation test of the three kinds of time registration method by using matlab. The results of the test prove the validity of the algorithm and compare the advantages and disadvantages of each algorithm. The conclusion has some reference function on the choice of time registration in practical engineering applications. In addition, because the existing time registration algorithms are mostly for a specific problem and specific conditions, the performance of different registration method to different target motion model is different. Therefore, to seek a registration method, which is common and can adapt to different motion model, is the direction of the next step.

\section{References}

[1] White F.A model for data fusion, in Proc.1st National Symposium Sensor Fusion, 1998,2: 149_ 158.

[2] Steinberg A N, Bowman C L, and White F E, Revisions to the JDL data fusionmodel, in AeroSense'99 International Society for Optics and Photonics, 1999:430-441

[3] B.Wang, and F.Li, The research on multiple targets tracking based on the data fusion technique, Journal of Xidian University(Natural Science), 25(3): 269-272, 1998

[4] Blair W D, Rice T R, Alouani A T, et al. Asynchronous data fusion for target tracking with a multitasking radar and optical sensor. Orlando, 91, Orlando, FL. International Society for Opticsand Photonics, 1991:234-245

[5] Y.Zhou, G.Shen, J.Fang, et al. Target tracking based on fusion of multi-sensor, Acta Aeronautica ET Astronautica Sinica, (5):536-540, 1998.

[6] K,Liang, P, Quan, G, Song, et al. The study of multi-sensor time registration method. Journal of Shaanxi University of Science and Technology(Natural Science Edition), 2006,24(6):111-114. 
[7] L.Liu, Post-Flight Data Processing of Trajectory Measurement. Beijing: National Defense Industry Press, 2000.

[8] W.Wang, Research on multi sensor spatial temporal registration. Electronic Science Research Institute, 2014.

[9] L.Shi, Research on time registration in multi sensor information fusion. National Defense Science and Technology University, 2010. 